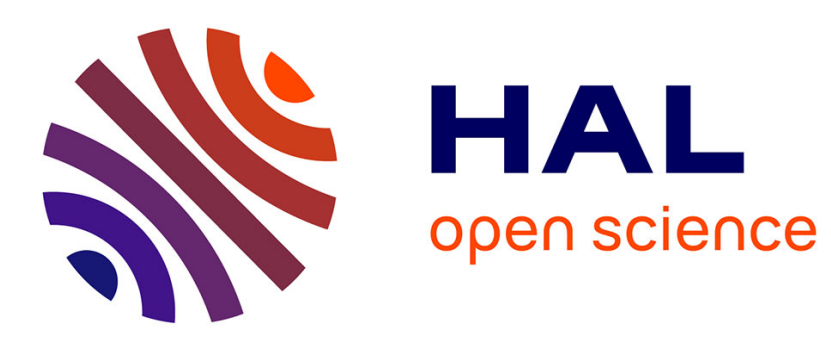

\title{
Quantitative Ultrasound Assessment of Cortical Bone Properties Beyond Bone Mineral Density
}

Q. Grimal, P. Laugier

\section{To cite this version:}

Q. Grimal, P. Laugier. Quantitative Ultrasound Assessment of Cortical Bone Properties Beyond Bone Mineral Density. Innovation and Research in BioMedical engineering, 2019, 40 (1), pp.16-24. 10.1016/j.irbm.2018.10.006 . hal-02328244

\section{HAL Id: hal-02328244 https://hal.sorbonne-universite.fr/hal-02328244}

Submitted on 23 Oct 2019

HAL is a multi-disciplinary open access archive for the deposit and dissemination of scientific research documents, whether they are published or not. The documents may come from teaching and research institutions in France or abroad, or from public or private research centers.
L'archive ouverte pluridisciplinaire HAL, est destinée au dépôt et à la diffusion de documents scientifiques de niveau recherche, publiés ou non, émanant des établissements d'enseignement et de recherche français ou étrangers, des laboratoires publics ou privés. 


\begin{tabular}{|c|}
\hline $\begin{array}{l}\text { Quantitative ultrasound assessment of cortical bone } \\
\text { properties beyond bone mineral density }\end{array}$ \\
\hline Q. Grimal $^{\mathrm{a}}$, P. Laugier ${ }^{\mathrm{a}}$ \\
\hline
\end{tabular}

6 Abstract

7

The development of quantitative ultrasound (QUS) technologies to measure bone is motivated by the need to overcome the limitations of X-ray based methods, measuring bone mineral density (BMD) which is the gold standard to date for the diagnosis of osteoporosis. Because it uses mechanical waves, the ultrasound modality is a particularly relevant means to probe bone mechanical resistance. The vast majority of QUS technologies commercialized to date merely aim to provide surrogate markers for BMD. During the past decade, innovative QUS approaches have emerged to assess bone beyond BMD. This may be achieved by (1) specifically assessing the cortical bone compartment, independently of trabecular bone, and (2) providing intrinsic bone properties such as cortical bone thickness and material properties. One specific motivation is to estimate intracortical porosity, a quantity reflected in material properties. This article aims at an overview of recent QUS developments to measure cortical bone properties. We also draw a picture of the current knowledge on bone material properties of interest for bone QUS. We discuss the potential of ultrasound to provide novel biomarkers of bone health through the assessment of material properties.

Keywords: ultrasound; cortical bone; elasticity; porosity; thickness; imaging; velocity

\section{Graphical abstract}

\section{Highlights}

- Bone fragility assessment would benefit from an accurate evaluation of cortical bone 


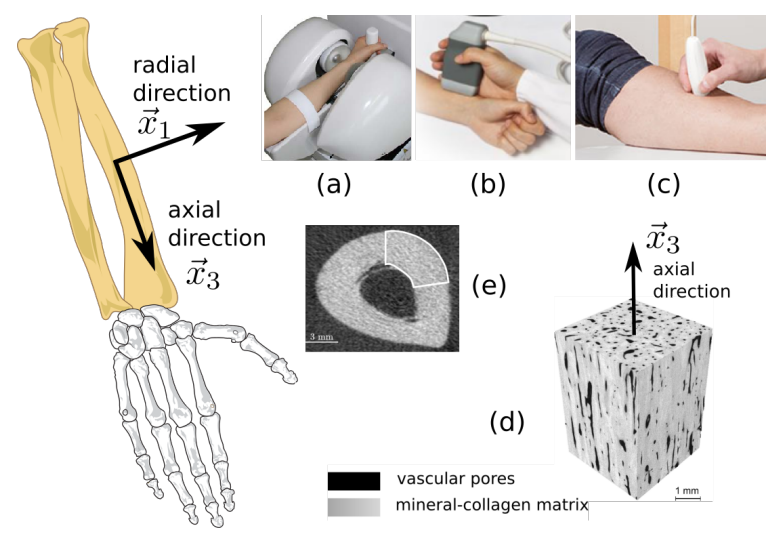

Figure 1: *

[Graphical abstract] Bone anatomy and QUS measurement configurations. (a) Measurement in bone radial direction with a through transmission approach (courtesy of OYO Electric CO., LTD., Japan); (b) Measurement in bone axial direction with bidirectional axial transmission to measure guided waves (courtesy of AZALEE, France) ; (c) Measurement in bone radial direction with a pulse-echo approach (courtesy of Bindex,

Finland) ; (d) image of cortical bone microanatomy obtained with synchrotron radiation microtomography showing the vascular porosity mainly oriented along the bone axis ; (e) X-ray image of a cross-section of the distal radius with a depicted region of interest for a typical QUS measurement with axial transmission.

- Innovative QUS technologies aim to measure intrinsic properties of cortical bone

- Available technologies measure bone thickness and bulk wave velocities

- Intracortical porosity, a fingerprint of remodeling, can be deduced from material properties

- Ex vivo documentation of material properties of pathological bone tissues is lacking

\section{Reference for citation}

Grimal Q, Laugier P. Quantitative ultrasound assessment of cortical bone properties beyond bone mineral density. IRBM. 2018 Volume: 40 Issue: 1 Pages: 16-24 


\section{Introduction}

Bone fragility associated to primary or secondary osteoporosis and the consequent risk of fracture is an important medical threat. Among the population aged over 50 years old, one in three women and one in five men will suffer a fracture associated to osteoporosis. Nine million fragility fractures occurred annually worldwide at the beginning of the 21th century [1]. Fracture risk prediction is assessed based on clinical factors and, in the standard approach, dual energy X-ray absorptiometry (DXA) in order to assess bone mineral density (BMD). However, it is well accepted that BMD assessed with DXA has strong limitations, in particular it has a lack of sensitivity $[2,3]$, and DXA is a ionizing method.

Our bones are comprised of two types of porous tissues: cortical bone is the dense tissue that forms the outer shell of the bones; trabecular, or spongious, bone is the more porous tissue partly filling the bones. While for several decades, bone alteration in osteoporosis has essentially been described to be trabecular bone loss [4], a focus has been placed in recent years on cortical bone which has been recognized to also play a key role in bone resistance in particular at fracture sites such as the proximal femur $[5,6]$ and the distal radius [7]. Aging is associated with an increased cortical porosity and thinning of the cortical shell. In old age, $70 \%$ of all appendicular bone loss may arise from the cortical compartment [8]. It follows that including an accurate evaluation of cortical bone in skeletal status assessment could improve diagnosis and treatment monitoring.

Cortical bone mechanical properties depend on the properties of the pore network (volume fraction of pores or, shortly, the porosity, and microarchitecture) and the properties of the extracellular mineralized matrix (shortly, matrix) surrounding pores. Pathologies, aging, and treatments may alter matrix material properties through modifications of collagen and mineral $[9,10,11,12]$ and the pore network $[13,8,11]$. In the last years, cortical bone porosity has been increasingly recognized as a fracture risk factor $[14,15,16]$.

Cortical bone thickness (CTh) is also a critical quantity for the stability of a bone during daily activities and in falls $[5,17]$. A reduction of $\mathrm{CTh}$ is usually associated to an increase of porosity [16].

Techniques to assess specifically the cortical compartment of the skeleton have been developed recently. These include X-ray computed tomography (CT) [18], indentation to specifically probe bone matrix [19], and quantitative ultrasound (QUS) methods. X-ray CT is extensively used for bone quantitative imaging, but essentially in clinical research. The most 
advanced X-ray CT technique is high-resolution peripheral computed tomography (HR-pQCT), a method available since 2004. It is a 3-D imaging technique, that allows a quantitative analysis of the cortical and trabecular bone compartments with a physical resolution of the order of $100 \mu \mathrm{m}$. It yields estimates of bone density, microarchitecture, and geometry. It can be used to measure CTh and, to some extent, porosity, at the distal radius and tibia. This modality, however, like conventional CT, will unlikely be used as a widespread diagnostic tool for osteoporosis due to cost issues and ionizing radiations. The focus of the present review is on bone QUS methods to measure cortical bone.

In the past three decades, researchers have developed QUS methods to measure trabecular and cortical bone $[20,21]$ motivated by the need to overcome the limitations of DXA and provide a non ionizing, portable, easily accessible, and affordable diagnostic tool for osteoporosis. The ultrasound modality is thought to be a particularly relevant means to probe bone health because it uses mechanical waves which are inherently sensitive to mechanical properties contributing to bone overall resistance.

One strategy in bone QUS research has long been to provide ultrasound variables, based on attenuation, velocity or backscatter measurements, as surrogate markers for BMD, the gold standard to date for the diagnosis of osteoporosis. The vast majority of bone QUS technologies commercialized and used in clinical studies since the 1990's fall into this category. This includes the earliest and best-validated clinical bone ultrasound devices [22] measuring in transmission the heel bone (mainly composed of trabecular bone), as well as recently developed pulse-echo techniques targeting different skeletal sites such as the spine and hip [23, 24]. The ultrasound surrogate for BMD predicts fracture risk, although, compared to DXA, it has not shown its superiority $[25,20]$ to date.

Another strategy in bone QUS research has been to measure intrinsic bone properties that convey information beyond BMD, such as CTh and material properties, such as bulk wave velocities. In that vein, approaches have been proposed during the past decade such as pulse-echo [26] and axial transmission [27] techniques which we cover in this review. The development of such QUS approaches requires a priori knowledge of physiological values of bone material properties and of their expected ranges variation in different pathologies.

This paper reviews the recent progress in QUS approaches aiming at the evaluation of bone fragility through the measurement of the thickness and material properties of cortical bone. We also draw a picture of the current knowledge on bone material properties of interest for in vivo ultrasound eval- 
uation. After this introduction as background, we review in section 2 cortical bone microanatomy and material properties. Section 3 presents QUS approaches to measure cortical bone. In Section 3.2, we account for the recent research aiming at a concurrent assessment of cortical bone anatomy and material properties. In Section 4, we discuss the potential of ultrasound to provide novel biomarkers of bone health through the assessment of material properties.

\section{Cortical bone tissue}

\subsection{Anatomy}

At its highest level of hierarchical organization, i.e., the millimeter (mm)scale, or mesoscale [28], cortical bone can be considered as a two-phase composite material: a mineralized collagen matrix pervaded by a porous network [29] (Fig. 2(d)). Bone mechanical properties are determined by the properties of the two phases: (1) pore structure and relative volume, and (2) matrix composition and microstructure.

At the nanometer scale, collagen molecules form fibrils which are progressively mineralized forming the elementary blocks of the matrix. Arrays of mineralized collagen fibrils assemble into lamellae to form cylindrical structures called osteons, which are the most prominent motifs at the highest microstructural level in cortical bone. In long bones, osteons align roughly parallel to the axis of the diaphysis (central tube-like part of long bones) and to the bone outer surface [30]. The vascular pore network is comprised of the roughly cylindrical Haversian canals (median size $\sim 50 \mu \mathrm{m}$ ) occupying the center of osteons, connected transversely by the Volkman's canals. Unbalanced bone remodeling occurring, e.g., in aging tend to change the porosity and the morphology of the pores. While pores occupy more or less $5 \%$ of the bone material volume before 40 years old, after 40 , differences are reported between women and men, the inter-individual variations increase, and, in women, the average porosity may reach about $15 \%[13,31]$. Also, porosity is known to be spatially heterogeneous within a bone, e.g., around the circumference and in the radial direction $[13,32,33]$.

\subsection{Notations}

Anatomical directions in a bone diaphysis are conveniently associated to a Cartesian reference frame where directions 1, 2, and 3 stand for radial, circumferential, and axial (along the diaphysis) directions (Fig. 2).

Ultrasound propagation in bone comprises the propagation of dilatational and shear waves and is affected by material anisotropy leading to a 


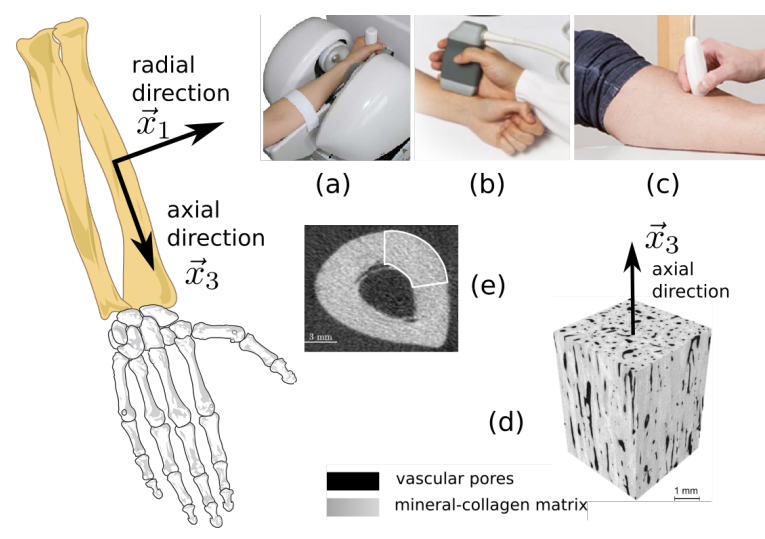

Figure 2: Bone anatomy and QUS measurement configurations. (a) Measurement in bone radial direction with a through transmission approach (courtesy of OYO Electric CO., LTD., Japan); (b) Measurement in bone axial direction with bidirectional axial transmission to measure guided waves (courtesy of AZALEE, France) ; (c) Measurement in bone radial direction with a pulse-echo approach (courtesy of Bindex, Finland) ; (d) image of cortical bone microanatomy obtained with synchrotron radiation microtomography showing the vascular porosity mainly oriented along the bone axis ; (e) X-ray image of a cross-section of the distal radius with a depicted region of interest for a typical QUS measurement with axial transmission.

direction-dependent speed of sound. Speed of sound is the square root of an elasticity-to-mass density ratio. We recall that the elasticity law may be written using Voigt notation as $\sigma_{i}=C_{i j} \epsilon_{j}$, where $\sigma_{i}$ and $\epsilon_{j}$ are components of the stress and srain vectors respectively, and $C_{i j}$ is the stiffness matrix. Stiffness constants $C_{i i}(i=1 \ldots 3)$ correspond to longitudinal loadings (of traction-compression type) along the different anatomical directions, $C_{i i}$ $(i=4 \ldots 6)$ are the shear moduli, and $C_{i j}=C_{j i}(i \neq j)$ correspond to mixed mode loadings. Engineering moduli, i.e., Young's moduli and Poisson ratios are defined as combinations of stiffness constants [34]. In the following, the bulk wave velocity (BWV) of dilatational waves, $\sqrt{C_{11} / \rho}$, and $\sqrt{C_{33} / \rho}$, where $\rho$ is the mass density, are denoted respectively radial BWV and axial BWV.

\subsection{Elastic anisotropy}

Cortical bone is most often described as an orthotropic material, that is, the material has a plane of symmetry associated to each anatomical direction. Such a material is characterized by nine distinct elastic moduli. Where orthotropy is weak as in the central portion of the diaphysis, a transversely isotropic material model, characterized by five moduli only [35, 36], 
is usually assumed. In long bones, the typical anisotropy ratio between longitudinal coefficients in the axial and radial (or circumferential) directions is between 1.3 and 2.5 [35, 37]. Anisotropy of cortical bone is due to both the preferential orientation of the vascular pores and the elastic anisotropy of the mineralized matrix due to the orientation of the mineralized collagen fibers. Theoretical studies have shown that bone mesoscale anisotropy mostly stems from the anisotropy of the matrix, the preferential orientation of the pores leading to moderate values of anisotropy $\left(C_{33} / C_{11} \sim 1.1-1.3\right.$ depending on the pore volume fraction) when the mineralized matrix is assumed to be isotropic $[38,39,40]$.

\subsection{Bone material properties}

A large number of ex vivo studies have reported elasticity values in cortical bone. The average Young's modulus along the diaphysis is typically around 14-20 GPa [41, 42, 37]. The average Young's modulus perpendicular to the diaphysis is around $11 \mathrm{GPa}$ [37] but is much less documented. The average shear modulus corresponding to a torsion experiment around an axis parallel to the diaphysis is about 4-6 GPa [42, 37].

The mass density of cortical bone ranges typically between 1.6 and $2 \mathrm{~g} . \mathrm{cm}^{-3}$. The variations of mass density are due to a combination of variations of the bone volume fraction in a volume of interest (i.e., the bone is more or less porous) and the variations of the mass density of the extracellular mineralized matrix. The latter are usually small because the volume fraction of mineral in mature bone remains relatively constant[43] . It follows that in practice mass density and porosity are highly correlated. \left. The above interval ([1.6-2] ${\mathrm{g} . \mathrm{cm}^{-3}}^{-3}\right)$ approximately corresponds to a range of porosity between $30 \%$ (extremely high porosity for cortical bone) and a few percents. In this range of density values, the elastic constants vary of $\pm 30-50 \%$ around their nominal values [37] (Fig. 3).

In a large number of ex vivo studies, BWVs in cortical bone specimens have been measured together with mass density in order to derive elasticity [44]. However, the BWVs values were not reported as such. Overall, compared to elasticity, BWV is much less documented. Eneh et al. [45] measured radial BWV ex vivo on parallelepiped samples of femoral bone from 18 donors and found $3202 \mathrm{~m} / \mathrm{s}( \pm 77)$ (Fig. 4). Lefevre et al. [46] measured radial and axial BWVs ex vivo in samples of fibula from 16 donors and found, respectively, $3137 \mathrm{~m} / \mathrm{s}( \pm 486)$ and $3994 \mathrm{~m} / \mathrm{s}( \pm 178)$. Grondin et al. [47], combining measurements in several quadrants of the femur of 4 donors found $3976 \mathrm{~m} / \mathrm{s}( \pm 72)$ for axial BWV. Mathieu et al. [48] investigated the radial variation of axial BWV in 11 femurs and found $3586 \mathrm{~m} / \mathrm{s}( \pm 255)$. In 


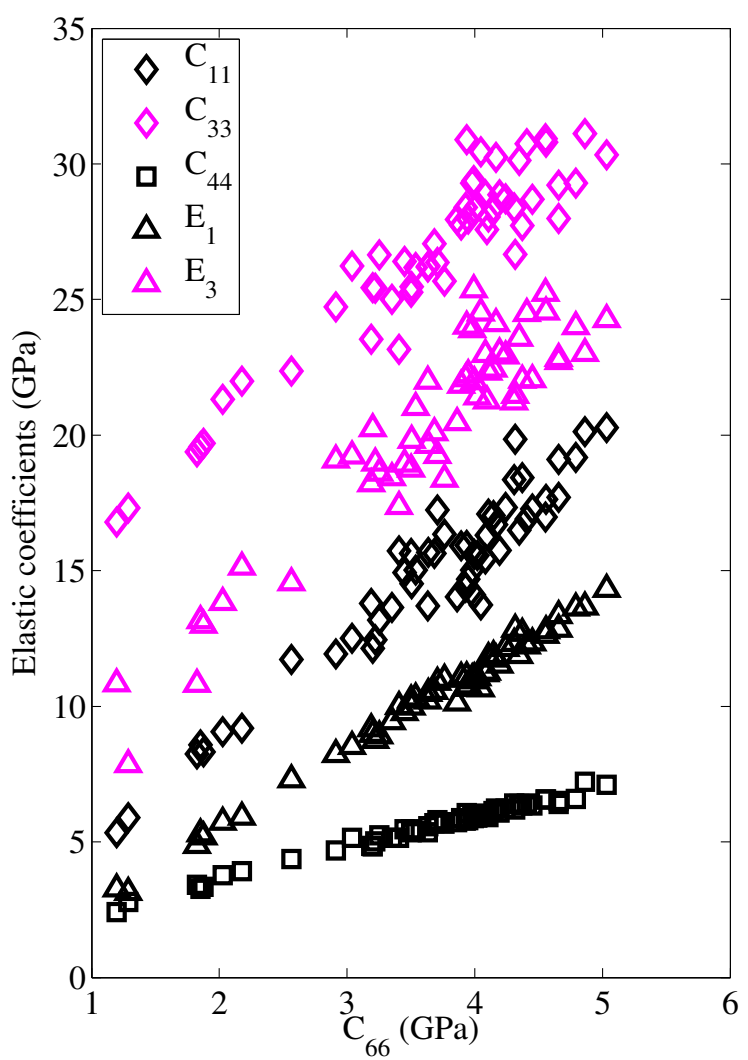

Figure 3: This figure, reproduced from [37], illustrates the ranges of variations of elastic coefficients (ex vivo measurements). Stiffness and engineering coefficients of 55 cortical bone specimens from the tibia, are plotted as a function of $C_{66}$ to illustrate the interdependency of the different elastic coefficients. The range of mass density values is [1.62.0] g.cm ${ }^{-3}$ for the different specimens. 
vivo, Greenfield et al. [49] combined radiographic measurement of radius thickness and pulse echo data to determine BWV in the radial direction, they found (mean \pm 1.5 standard deviation) $3311 \mathrm{~m} / \mathrm{s}( \pm 307)$ in men and $3359 \mathrm{~m} / \mathrm{s}( \pm 297)$ in women.

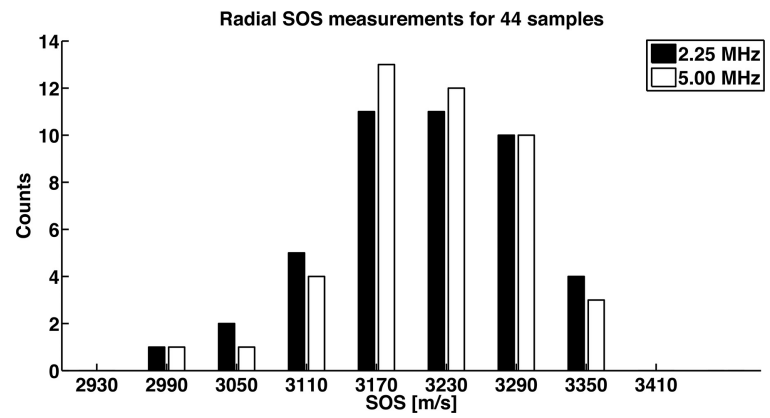

Figure 4: Reproduced from [45]. Histogram (numbers represent the center of bins that are $60 \mathrm{~m} / \mathrm{s}$ in width) of the average values of radial bulk wave velocity (denoted SOS for 'speed of sound' in the figure) in 44 cortical bone samples from the femur diaphysis. The black and white bars represent radial BWV measured at 2.25 and $5.00 \mathrm{MHz}$, respectively.

As explained in sec 3, the design of future bone QUS methods to measure material properties rely on reference data at the typical measurement sites (radius, tibia). Some ex vivo studies have provided values for cortical bone material properties such as elasticity, density and BWVs. However, the large majority of this data was obtained from the diaphysis of the femur, which is not a site measured with QUS, and, to a lesser extent, from the tibia. Little data exists on radius bone due to the difficulty to measure small samples.

The available data was obtained on cadaveric bones, usually from elderly donors without documentation on the existence of bone pathologies. During childhood, there is a well documented effect of age on bone mineral density [50]. The changes over age of material properties are not covered in the present review, although a bone QUS method can be dedicated to measure children's bones.

To conclude, elastic properties and BWVs at radius and tibia need to be better documented. In particular, it is not clear whether or not bone material has distinct characteristics at these two sites (tibia, as opposed to radius, is a weight-bearing bone) and if these sites are comparable to the femur for which much more data is available. A better documentation of the tibia and radius bones may be obtained with resonant ultrasound spectroscopy (RUS) [51, 52] a technique that allows retrieving the full stiffness tensor from small-sized rectangular parallelepiped specimens. 


\section{Cortical bone quantitative ultrasound}

We review below cortical bone QUS approaches aiming at the measurement of cortical bone thickness (CTh) and material properties. These QUS approaches are essentially developed for the radius and tibia [53] because these sites are easily accessible to ultrasound. The radius, but not the tibia, is an osteoporotic fracture site. Osteoporosis is a systemic disease, accordingly, measuring bone at any site is expected to have a clinical value, which has been proven with several approaches implemented at the heel, phalanx, tibia and radius.

In general, the measurement of $\mathrm{CTh}$ with ultrasound in vivo relies on an a priori knowledge, or a joined measurement, of one or several material properties. When measuring bone in the radial direction with a pulse-echo method (Fig. 2(c)), the ultrasound raw data, in terms of time-of-flight, couple information on material properties (BWVs) and anatomy (CTh). With the axial transmission method, measuring the propagation of guided waves in the cortical envelope of the diaphysis (Fig. 2(b)), information on bulk wave velocities and CTh are also coupled (except in the limit case of a sufficiently large CTh and high frequency, in which case a lateral wave can be measured [54], giving access directly to the axial BWV without requiring the knowledge of thickness).

At QUS measurement sites, CTh shows a large range of values across individuals: typically 1 to $4 \mathrm{~mm}$ in radius and 1.5 to $5 \mathrm{~mm}$ in tibia $[26,55]$. In contrast, the range of variations of material properties is smaller, e.g., typically, the mass density varies between 1.8 and $2 \mathrm{~g} . \mathrm{cm}^{-3}$, and the BVWs and elastic coefficients may respectively vary of $\pm 10 \%$ and $\pm 50 \%$ around an average value. Because it is intrinsically difficult to retrieve concurrently several bone characteristics (namely CTh and material properties), to date, clinical implementations of QUS approaches only provide CTh assuming fixed values of material properties. Several approaches are under development to overcome this limitation.

\subsection{Current approaches}

Karjalainen et al. [26], following Greenfield et al. [49] and Wear [56] implemented a pulse-echo method (Fig. 2(c)) to measure CTh at the tibia and radius using a fixed value of the radial $\mathrm{BWV}(3565 \mathrm{~m} / \mathrm{s})$. This value was chosen such that CTh determined from the QUS measurement matches the reference CTh obtained from HR-pQCT in healthy volunteers.

Otani et al. developed a method to measure the distal radius in throughtransmission, i.e., the wrist is placed between a pair of confocally aligned 
transducers, [57] (Fig. 2(a)). Ultrasound passes through both the lateral and medial cortical layers and through the trabecular bone in the metaphysis. Assuming a layered model of bone and some fixed material properties (radial BWV in cortical bone is set to $3300 \mathrm{~m} / \mathrm{s}$ ), the method yields, among other parameters, the sum of the cortical thicknesses at the inlet and outlet sides of the the US beam.

So-called axial transmission measurements involve guided waves propagating in the cortical layer in the direction of the bone axis (Fig. 2(b)). Guided waves propagation is highly sensitive to variations of CTh [27, 58]. The waveguide thickness is retrieved by resorting to an optimization algorithm to solve an inverse problem. Precisely, the cortical bone layer is modeled as a plate of given (fixed) material properties and unknown thickness (CTh), to be determined by fitting the simulated ultrasonic behavior of the plate to experimental data. Moilanen et al. [59] demonstrated on ex vivo radii that $\mathrm{CTh}$ can be retrieved from the signal of a $200 \mathrm{kHz}$ guided wave; in this work, bone material was assumed isotropic with fixed properties $(B W V=4000 \mathrm{~m} / \mathrm{s})$. In a subsequent study, Vallet et al. [55] exploited several guided modes [60] to retrieve CTh using US signals centered at $1 \mathrm{MHz}$ and transverse isotropic fixed properties (radial BWV=3024 m/s; axial $\mathrm{BWV}=3753 \mathrm{~m} / \mathrm{s}$ ).

During the last few years, the first clinical studies with the above approaches have been conducted. CTh was found to be different in fractured versus non fractured patients, all with impaired kidney function [61]. Sai et al. [57] observed the expected decrease of CTh with age in an healthy population and the higher thickness of males compared to females. The pulse echo method of Karjalainen et al. [26], combining measurement of CTh and patient's characteristics, was shown to predict femoral neck BMD with good accuracy [62] and to discriminate patients with hip osteoporosis from controls [63, 24].

\subsection{Future of cortical bone QUS}

In all the above-mentioned approaches, cortical bone material properties are assumed to be identical for all subjects. This a limitation as tissue properties may vary between individuals and between sites (section 2). Not only this likely impairs the accuracy of the determination of CTh but also material properties themselves may give a valuable additional information on bone quality. In particular, material properties are strongly related to porosity, which is a recognized fracture risk factor $[64,15]$ (see sec. 4). 


\subsubsection{Innovative methods}

One perspective for cortical bone QUS, based on existing approaches, is to couple the determination of CTh and that of material properties. This can be achieved with several approaches. In pulse-echo mode, methods using a transfer function approach $[65,66,67]$ could in principle be designed to retrieve BWVs, density, attenuation, and CTh exploiting the times-of-flight and amplitudes in the reflected signals. The multimode axial transmission technique allows retrieving $\mathrm{CTh}$ and material properties or porosity, which has been demonstrated ex vivo [68, 69, 70].

Obtaining ultrasound images of the internal structure of cortical bone is another exciting perspective. Conventional ultrasound scanners are used in clinical practice to image the outer surface of bones allowing for the diagnosis of bone fractures [71]. However, these ultrasound systems fail to reveal the internal structure of bones because (1) the algorithm used to construct the image assumes that ultrasound follow a straight path and that BWVs do not vary along the path; (2) attenuation in cortical bone is relatively large; and (3) important energy loss occur at the soft tissue-bone interface caused by the large acoustic impedance mismatch. Using wave scattering theory to model the wave path, Zheng et al. [72] obtained ex vivo an image of the cortical layer of a bovine femur. Taking advantage of the tremendous performance improvement of hardware electronics in ultrasound scanners in the last years and developing a dedicated image reconstruction technique, Renaud et al. [73] have recently obtained in vivo quantitative images of the cortical layer of human radius and tibia (Fig. 5). The velocity of bulk dilatational waves in the different anatomical directions is recovered by combining a measurement of the lateral wave and optimizing image quality.

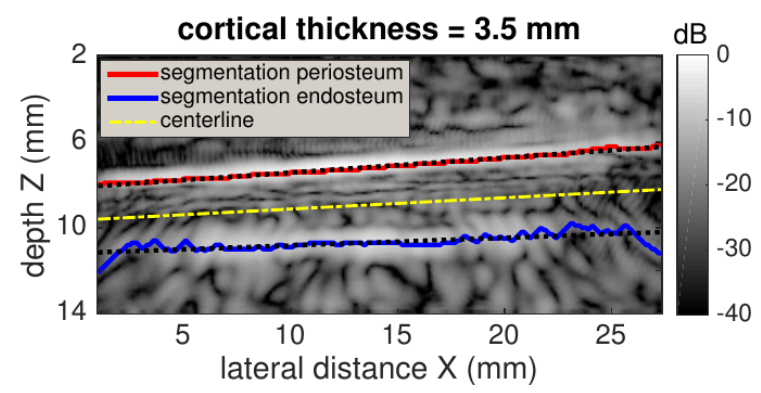

Figure 5: Reproduced from [73]. Ultrasound image of the cortical layer of a radius in vivo. Red and blue lines correspond to the periosteum and endosteum. Straight lines (doted black line) approximate the interfaces. The cortical thickness, defined as the mean distance between these lines is found to be $3.5 \mathrm{~mm}$ for this acquisition and is in agreement with the thickness measured with HR-pQCT. 
Lasaygues et al. have proposed to reconstruct a quantitative image of an entire transverse cross-section of a long bone with a tomography setup, using scattering theory and Born approximation [74] or using a full waveform approach [75].

\subsubsection{Models of material properties for cortical QUS}

Depending on the QUS measurement approach and the type of waves involved (shear and dilatational waves in different anatomical directions), more or less material parameters are involved in the processing of ultrasound signals. While only the radial dilatational BWV is involved in pulse-echo methods, no less than four material parameters may be involved in axial transmission configuration (e.g., one BWV and three elastic anisotropy ratios [68] ). The latter parameters are the quantities directly accessible from a measurement. In the methods implemented for clinical applications, nor mass density nor elastic coefficients can be inferred without resorting to a model of bone material properties (e.g.,[40] ) relating these quantities and those directly accessible from a measurement.

A priori knowledge of bone material properties is mandatory to solve the coupled problem of the determination of $\mathrm{CTh}$ and material properties. Such information is all the more important that the number of parameters to retrieve is large. It has been pointed out that the different elastic coefficients are correlated (Fig. 3) and strongly depend on density [76, 41, 77, 37, 52], and porosity [36] (see also sec. 4). As a consequence, a simplified model of cortical bone elastic properties with a limited number of parameters $[78,79]$ could be used in order to reduce the number of unknowns when solving the QUS inverse problem. Such an approach was implemented by Bochud et al. [69] where cortical bone was modeled as a pore network of variable porosity embedded in a matrix with fixed elastic properties [40, 80] (an implementation of the model is available online [81]; the model predictions are plotted against ex vivo elasticity measurements in Fig. 6).

\section{Measuring material properties as potential biomarkers of bone health}

Managing bone health often starts by assessing the risk of fracture of an individual. This depends on many factors related to the risk of an individual to fall, and to the ability of a bone to resist a low trauma. The latter depends both on bone size and geometry, and material properties. In this section, we briefly review the relationships between, on the one hand, material properties that may be derived in vivo from QUS measurements 
and, on the other hand, strength, fracture risk factors, and quality of the extracellular matrix.

\subsection{Bone resistance}

As mentioned in the introduction, one potential advantage of ultrasound over X-ray approaches is to assess material properties beyond the mere quantity of bone reflected in BMD measured with an X-ray based technique.

Bone resistance to fracture is typically characterized by strength (i.e., ultimate stress before rupture) and toughness (i.e., resistance to crack propagation) [82]. It is yet unclear to which extent ultrasound, probing bone at very small strains in a linear regime, may yield information on bone resistance.

Correlations have been reported between bone resistance and elastic properties as for engineering and natural materials in general [83]. Indeed, post-yield and elastic properties are all determined by the biochemical composition and the microstructure of bone. It is thus expected that elasticity and BWVs reflect material resistance to some extent. Pooling results of compression testing of children and adult bone, Öhman et al. [84] found a high correlation between yield stress and Young's modulus $\left(R^{2}=0.88\right)$. Weaker correlations have been found in studies considering only specimens from adult donors $\left(R^{2}=0.25\right.$ in three-point bending tests [85]; $R^{2}=0.53$ and 0.56 in tension and compression tests, respectively [41]). Further studies should elucidate more precisely how much of bone material resistance (strength or toughness) can be learned from the measurement of elastic properties and BWVs.

\subsection{Elasticity and bulk wave velocities reflect porosity and matrix properties}

Porosity, a fracture risk factor [15], is an important determinant of stiffness variations $[86,87]$. Several authors have reported correlations between porosity and elastic moduli, although the range of correlation coefficients is quite large. For example, Mirzaali et al. [42] found $R^{2}=0.08$ (Young's modulus) and $R^{2}=0.47$ (shear modulus), Granke et al. [36] and Cai et al. [88] found $R^{2}$ in the range $0.70-0.84$ for all shear and longitudinal stiffness coefficients. Relatively large relative variations of stiffness in the porosity range [2.9-26.9\%] were reported [36]: $58 \%, 34 \%, 48 \%$, and $59 \%$ for $C_{11}, C_{33}$, $C_{44}$, and $C_{66}$, respectively (Fig. 6). Overall, as porosity increases, $C_{11}$ (longitudinal elasticity transverse to Haversian canals) decreases more compared to $C_{33}$ (longitudinal elasticity in the direction of Haversian canals). This is consistent with results of theoretical studies [89, 79, 40]. 
Data suggest that porosity is also a strong determinant of BWVs. In axial direction, porosity was found to explain about $30 \%$ of BWV variation $[47,48]$. In radial direction it was found to explains about $50 \%$ of BWV variations [45].
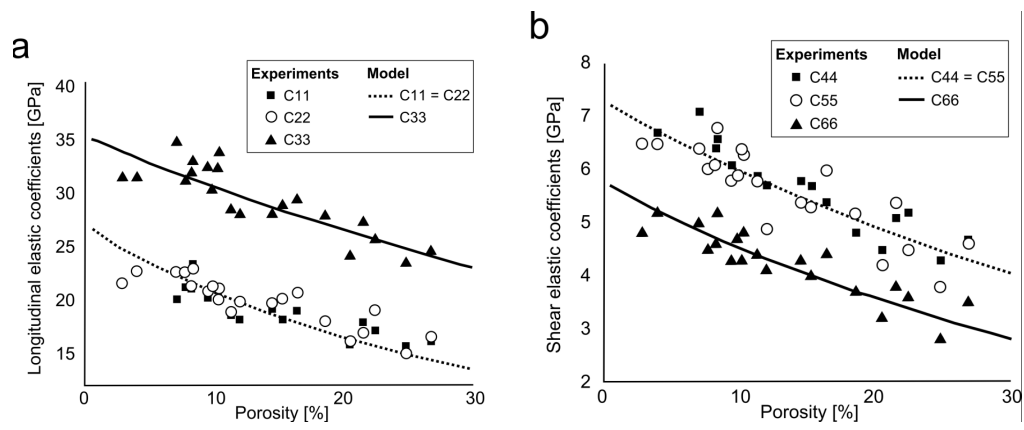

Figure 6: Reproduced from [36]. (a) Longitudinal and (b) shear mesoscopic elastic coefficients versus porosity in 21 cortical bone samples from the femur. The model based on continuum mechanics laws (solid and dotted lines), assuming fixed matrix properties and variable porosity, predicts the trend of variation of elasticity.

There is few data suggesting that variations of material properties of the matrix may be reflected in QUS signals in human bones [90]. There is, however, ex vivo data on prepared specimens pointing at an effect of matrix properties variations on mesoscale elasticity. For instance, Rho et al. [91] found that variations of the mesoscale axial Young's modulus $\left(R^{2}=0.49\right)$ correlated to the variations of the matrix elasticity (probed with nanoindentation). Granke et al.[36] found that mesoscale elasticity was correlated with matrix acoustical impedance (a proxy for stiffness) probed with acoustic microscopy $\left(R^{2}<0.25\right)$. In another study, variations of matrix impedance has been found to explain as much as $52 \%$ of axial BWV [47]. Eneh et al. [92] showed, with simulations performed on a limited number of samples, that the correlation of BWV in the radial direction with porosity may be lost due to inter-individual variations of matrix properties.

The main determinant of matrix stiffness variations is commonly thought to be the mineral content. This is well evidenced considering a large variety of bone samples taken from different species [86], and theoretical calculations predict that a change of $10 \%$ of mineral volume fraction leads to a change of typically more than $20 \%$ of matrix elastic coefficients [93, 94, 78]. In a recent study on femoral bone specimens from 19 elderly donors, Cai [88] found that more than $50 \%$ of mesoscale elasticity variations were associated to variations of mineral content. Collagen fibers mechanical quality 
and organizational patterns have also been proposed as possible determinants of mesoscopic properties. As far as we are aware, there is no data for human bone showing an effect of a pathological alteration of collagen on mesoscale elastic properties. However, artificial degradation of the collagen with chemical treatments is known to alter elastic properties [95].

From a mechanical standpoint, mesoscale cortical bone material properties such as density, elasticity, or BWVs are fully determined by the properties of the pore network and of the matrix. Whether or not a change of these properties is reflected at the mesoscale in a given dataset critically depends on the range of variations of the properties at the different scales. The data reported above was obtained from the tissues of donors with no documented medical history, hence the conclusions drawn from these studies only pertain to this type of population and cannot be extrapolated to groups of subjects carrying specific bone diseases. To conclude, for these bones from non-targeted populations, the available data suggest that a large part of the elasticity and BWVs variations is explained by the variation of porosity. This is consistent with the prediction of theoretical models which assume that the matrix properties have limited inter-individual variations and that porosity varies in a relatively large interval $[78,80]$ (sec. 3.2.2). Variations of the properties of the matrix (mineral content, impedance, elasticity) also impact mesoscale elastic properties and BWVs, however, only limited data is available. Some pathologies involving a low mineral content or a weak alignment of collagen fibers are expected to strongly affect mesoscale properties through modifications of both the porosity and the matrix. This calls for more studies designed to investigate the variations of BWVs and elasticity in different targeted populations.

\subsection{Biomarkers of material heterogeneity}

Aging may be associated to an increase of the heterogeneity of the distribution and size of the pores in the cortical bone layer, resulting in a gradient of porosity: high porosity close to the marrow and relatively low porosity close to the external surface of the bone [8] . In terms of mechanics of materials, this raises the question of the existence of a representative volume element of cortical bone material[28]. If the local variations of porosity are too strong, the cortical bone material can not be evaluated per se and the cortex needs to be considered as a structure. In case of a mild heterogeneity of porosity, it may be relevant to model the cortex material as a heterogeneous field of material properties. This issue has in part been theoretically addressed in an axial transmission QUS configuration [96, 97] but has not been implemented in clinical practice as far as we know. Since the 
heterogeneity of material properties within the cortex is expectedly associated to a reduced mechanical competence, it could be interesting to develop ultrasound biomarkers reflecting heterogeneity.

\section{Conclusion}

QUS technologies to measure cortical bone thickness, a proven biomarker of bone health, are available and used in vivo. Improvements of these technologies and disruptive technologies are expected to be available in a near future, which will achieve a coupled assessment of cortical thickness and material properties. One motivation is to estimate intracortical porosity, a quantity hardly directly measurable in vivo. Assessing porosity with ultrasound would be a significant progress because porosity is a recognized fracture risk factor and because it is a fingerprint of the remodeling activity. One route to infer porosity is to use empirical relationships, or material models, relating quantities measured with QUS and porosity. Other routes are currently being explored such as imaging blood perfusion using ultrasound contrast agent [98] and measuring ultrasonic attenuation assuming it has a strong relationship with pore properties [99].

Probing the quality of the mineralized collagen matrix in vivo with ultrasound is a far-reaching goal. It may be a reasonable objective in targeted pathologies providing that alterations of porosity and matrix properties are well documented ex vivo. Such documentation of acoustical properties in bone tissue with different pathologies is a keystone of the future development of bone QUS methods.

\section{Acknowledgment}

[1] C Cooper and F Ferrari. IOF Compendium of Osteoporosis. Technical report, International Osteoporosis Foundation, 2017.

[2] E S Siris, Y T Chen, T A Abbott, E Barrett-Connor, P D Miller, $\mathrm{L} \mathrm{E}$ Wehren, and M L Berger. Bone mineral density thresholds for pharmacological intervention to prevent fractures. Archives of Internal Medicine, 164(10):1108-1112, 2004.

[3] Karine Briot, Simon Paternotte, Sami Kolta, Richard Eastell, Dieter Felsenberg, David M. Reid, Claus C. Glüer, and Christian Roux. FRAX $($ : Prediction of major osteoporotic fractures in women from the general population: The OPUS study. PLoS ONE, 8(12), 2013. 
[4] A. M. Parfitt. Misconceptions (2): Turnover is always higher in cancellous than in cortical bone. Bone, 30(6):807-809, 2002.

[5] Paul M Mayhew, C David Thomas, John G Clement, Nigel Loveridge, Thomas J Beck, William Bonfield, Chris J Burgoyne, and Jonathan Reeve. Relation between age, femoral neck cortical stability, and hip fracture risk. The Lancet, 366(9480):129-135, 2005.

[6] Gerold Holzer, Gobert von Skrbensky, Lukas A Holzer, and Wolfgang Pichl. Hip fractures and the contribution of cortical versus trabecular bone to femoral neck strength. J Bone Miner Res, 24(3):468-474, mar 2009.

[7] P Augat, H Reeb, and L E Claes. Prediction of fracture load at different skeletal sites by geometric properties of the cortical shell. J Bone Miner Res, 11(9):1356-1363, 1996.

[8] R M D Zebaze, A Ghasem-Zadeh, A Bohte, S Iuliano-Burns, M Mirams, R I Price, E J Mackie, and E Seeman. Intracortical remodelling and porosity in the distal radius and post-mortem femurs of women: a crosssectional study. Lancet, 375(9727):1729-1736, 2010.

[9] Patrick Ammann, Isabelle Badoud, Sébastien Barraud, Romain Dayer, and René Rizzoli. Strontium ranelate treatment improves trabecular and cortical intrinsic bone tissue quality, a determinant of bone strength. Journal of Bone and Mineral Research, 22(9):1419-1425, 2007.

[10] Yohann Bala, Baptiste Depalle, Delphine Farlay, Thierry Douillard, Sylvain Meille, Helene Follet, Roland Chapurlat, J??r??me Chevalier, and Georges Boivin. Bone micromechanical properties are compromised during long-term alendronate therapy independently of mineralization. Journal of Bone and Mineral Research, 27(4):825-834, 2012.

[11] Laurianne Imbert, Jean-Charles Aurégan, Kélig Pernelle, and Thierry Hoc. Mechanical and mineral properties of osteogenesis imperfecta human bones at the tissue level. Bone, 65:18-24, 2014.

[12] Jirko Kühnisch, Jong Seto, Claudia Lange, Susanne Schrof, Sabine Stumpp, Karolina Kobus, Julia Grohmann, Nadine Kossler, Peter Varga, Monika Osswald, Denise Emmerich, Sigrid Tinschert, Falk Thielemann, Georg Duda, Wenke Seifert, Thaqif El Khassawna, David A. Stevenson, Florent Elefteriou, Uwe Kornak, Kay Raum, Peter 
Fratzl, Stefan Mundlos, and Mateusz Kolanczyk. Multiscale, converging defects of macro-porosity, microstructure and matrix mineralization impact long bone fragility in NF1. PLoS ONE, 9(1):1-12, 2014.

[13] V Bousson, A Meunier, Bergot C., E Vicaut, M A Rocha, M H Morais, A M Laval-Jeantet, and J D Laredo. Distribution of intracortical porosity in human midfemoral cortex by age and gender. Journal of Bone and Mineral Research, 16:1308-1317, 2001.

[14] L. A. Ahmed, R. Shigdel, R. M. Joakimsen, O. P. Eldevik, E. F. Eriksen, A. Ghasem-Zadeh, Y. Bala, R. Zebaze, E. Seeman, and Bjørnerem. Measurement of cortical porosity of the proximal femur improves identification of women with nonvertebral fragility fractures. Osteoporosis International, 26(8):2137-2146, 2015.

[15] Yohann Bala, Roger Zebaze, and Ego Seeman. Role of cortical bone in bone fragility. Current Opinion in Rheumatology, 27(4):406-413, 2015.

[16] Roger Zebaze, Cesar Libanati, Michael R. McClung, José R. Zanchetta, David L. Kendler, Arne Høiseth, Andrea Wang, Ali Ghasem-Zadeh, and Ego Seeman. Denosumab Reduces Cortical Porosity of the Proximal Femoral Shaft in Postmenopausal Women With Osteoporosis. Journal of Bone and Mineral Research, 31(10):1827-1834, 2016.

[17] Kyle K. Nishiyama, Heather M. Macdonald, Helen R. Buie, David A. Hanley, and Steven K. Boyd. Postmenopausal women with osteopenia have higher cortical porosity and thinner cortices at the distal radius and tibia than women with normal aBMD: An in vivo HR-pQCT study. Journal of Bone and Mineral Research, 25(4):882-890, 2010.

[18] Piet Geusens, Roland Chapurlat, Georg Schett, Ali Ghasem-Zadeh, Ego Seeman, Joost De Jong, and Joop Van Den Bergh. High-resolution in vivo imaging of bone and joints: A window to microarchitecture, may 2014.

[19] Adolfo Diez-Perez, Roberto Güerri, Xavier Nogues, Enric Cáceres, Maria Jesus Peñ, Leonardo Mellibovsky, Connor Randal, Daniel Bridges, James C. Weaver, Alexander Proctor, Davis Brimer, Kurt J. Koester, Robert O. Ritchie, and Paul K. Hansma. Microindentation for in vivo measurement of bone tissue mechanical properties in humans. Journal of Bone and Mineral Research, 25(8):1877-1885, 2010. 
[20] P Laugier. Instrumentation for in vivo ultrasonic characterization of bone strength. IEEE Trans Ultrason Ferroelectr Freq Control, 55(6):1179-1196, 2008.

[21] Kay Raum, Quentin Grimal, Peter Varga, Reinhard Barkmann, Claus C Glüer, and Pascal Laugier. Ultrasound to assess bone quality. Curr Osteoporos Rep, 12(2):154-162, 2014.

[22] E. V. McCloskey, J. A. Kanis, A. Odén, N. C. Harvey, D. Bauer, J. González-Macias, D. Hans, S. Kaptoge, M. A. Krieg, T. Kwok, F. Marin, A. Moayyeri, E. Orwoll, C. Gluër, and H. Johansson. Predictive ability of heel quantitative ultrasound for incident fractures: an individual-level meta-analysis. Osteoporosis International, 26(7):19791987, 2015.

[23] Sergio Casciaro, Francesco Conversano, Paola Pisani, and Maurizio Muratore. New perspectives in echographic diagnosis of osteoporosis on hip and spine. Clinical Cases in Mineral and Bone Metabolism, 12(2):142150, 2015.

[24] J. P. Karjalainen, O. Riekkinen, and H. Kröger. Pulse-echo ultrasound method for detection of post-menopausal women with osteoporotic BMD. Osteoporosis International, pages 1-7, 2018.

[25] Claus C. Glüer. A new quality of bone ultrasound research. IEEE Transactions on Ultrasonics, Ferroelectrics, and Frequency Control, 55(7):1524-1528, 2008.

[26] Jarme Karjalainen, Ossi Riekkinen, Juha Töyräs, Heikki Kröger, and Jukka Jurvelin. Ultrasonic assessment of cortical bone thickness in vitro and in vivo. IEEE Transactions on Ultrasonics, Ferroelectrics, and Frequency Control, 55(10):2191-2197, 2008.

[27] Petro Moilanen. Ultrasonic guided waves in bone. IEEE Transactions on Ultrasonics, Ferroelectrics, and Frequency Control, 55(6):1277-1286, 2008.

[28] Quentin Grimal, Kay Raum, Alf Gerisch, and Pascal Laugier. A determination of the minimum sizes of representative volume elements for the prediction of cortical bone elastic properties. Biomech Model Mechanobiol, 10(6):925-937, 2011. 
[29] D. M.L. Cooper, C. E. Kawalilak, K. Harrison, B. D. Johnston, and J. D. Johnston. Cortical Bone Porosity: What Is It, Why Is It Important, and How Can We Detect It? Current Osteoporosis Reports, 14(5):187-198, 2016.

[30] M Petrtyl, J Hert, and P Fiala. Spatial organization of the haversian bone in man. Journal of Biomechanics, 29(2):161-167, 1996.

[31] C D Thomas, S A Feik, and J G Clement. Increase in pore area, and not pore density, is the main determinant in the development of porosity in human cortical bone. J Anat., 209(2):219-30., 2006.

[32] M K H Malo, D Rohrbach, H Isaksson, J Töyräs, J S Jurvelin, I S Tamminen, H Kröger, and K Raum. Longitudinal elastic properties and porosity of cortical bone tissue vary with age in human proximal femur. Bone, 53(2):451-458, jan 2013.

[33] Egon Perilli, Yohann Bala, Roger Zebaze, Karen J Reynolds, and Ego Seeman. Regional Heterogeneity in the Configuration of the Intracortical Canals of the Femoral Shaft. Calcif Tissue Int, 97(4):327-335, oct 2015 .

[34] S C Cowin. Bone mechanics handbook, volume 1. CRC Press, Boca Raton, FL, second edition, 2001.

[35] David J Rudy, Justin M Deuerling, Alejandro A Espinoza Orías, and Ryan K Roeder. Anatomic variation in the elastic inhomogeneity and anisotropy of human femoral cortical bone tissue is consistent across multiple donors. J Biomech, 44(9):1817-1820, 2011.

[36] Mathilde Granke, Quentin Grimal, Amena Saïed, Pierre Nauleau, Françoise Peyrin, and Pascal Laugier. Change in porosity is the major determinant of the variation of cortical bone elasticity at the millimeter scale in aged women. Bone, 49(5):1020-1026, 2011.

[37] Simon Bernard, Joannes Schneider, Peter Varga, Pascal Laugier, Kay Raum, and Quentin Grimal. Elasticity-density and viscoelasticitydensity relationships at the tibia mid-diaphysis assessed from resonant ultrasound spectroscopy measurements. Biomech Model Mechanobiol, 15(1):97-109, 2016.

[38] Q Grimal, K Raum, A Gerisch, and P Laugier. Derivation of the mesoscopic elasticity tensor of cortical bone from quantitative impedance 
images at the micron scale. Computer Methods in Biomechanics and Biomedical Engineering, 11(2):147-157, 2008.

[39] Andrew P Baumann, Justin M Deuerling, David J Rudy, Glen L Niebur, and Ryan K Roeder. The relative influence of apatite crystal orientations and intracortical porosity on the elastic anisotropy of human cortical bone. J Biomech, 45(16):2743-2749, nov 2012.

[40] William J Parnell, M B Vu, Q Grimal, and S Naili. Analytical methods to determine the effective mesoscopic and macroscopic elastic properties of cortical bone. Biomech Model Mechanobiol, 11:883-901, nov 2012.

[41] L Duchemin, V Bousson, C Raossanaly, C Bergot, J D Laredo, W Skalli, and D Mitton. Prediction of mechanical properties of cortical bone by quantitative computed tomography. Med Eng Phys, 30(3):321-328, 2008.

[42] Mohammad J. Mirzaali, J. Jakob Schwiedrzik, Suwanwadee Thaiwichai, James P. Best, Johann Michler, Philippe K. Zysset, and Uwe Wolfram. Mechanical properties of cortical bone and their relationships with age, gender, composition and microindentation properties in the elderly. Bone, 93:196-211, 2016.

[43] P Roschger, E P Paschalis, P Fratzl, and K Klaushofer. Bone mineralization density distribution in health and disease. Bone, 42(3):456-466, mar 2008.

[44] R B Ashman, S C Cowin, W C van Buskirk, and J C Rice. A continuous wave technique for the measurement of the elastic properties of cortical bone. Journal of Biomechanics, 17(5):349-361, 1984.

[45] C. T.M. Eneh, M. K.H. Malo, J. P. Karjalainen, J. Liukkonen, J. Töyräs, and J. S. Jurvelin. Effect of porosity, tissue density, and mechanical properties on radial sound speed in human cortical bone. Medical Physics, 43(5):2030-2039, 2016.

[46] Emmanuelle Lefèvre, Philippe Lasaygues, Cécile Baron, Cédric Payan, Franck Launay, Hélène Follet, and Martine Pithioux. Analyzing the anisotropic Hooke[U+05F3]s law for children [U+05F3]s cortical bone. J Mech Behav Biomed Mater, 49:370-377, sep 2015.

[47] Julien Grondin, Quentin Grimal, Kazufumi Yamamoto, Mami Matsukawa, Amena Saïed, and Pascal Laugier. Relative contributions of 
porosity and mineralized matrix properties to the bulk axial ultrasonic wave velocity in human cortical bone. Ultrasonics, 52(4):467-471, 2012.

[48] Vincent Mathieu, Christine Chappard, Romain Vayron, Adrien Michel, and Guillaume Haïat. Radial anatomic variation of ultrasonic velocity in human cortical bone. Ultrasound in Medicine and Biology, 39(11):2185-2193, 2013.

[49] M Greenfield, J Duncan Craven, Alan Huddleston, Mary Kehrer, David Wishko, and Richard Stern. Measurement of the Velocity of Ultrasound in Human Cortical. radiation physics, 138:701-710, 1981.

[50] Adam D G Baxter-Jones, Robert A Faulkner, Mark R Forwood, Robert L Mirwald, and Donald A Bailey. Bone mineral accrual from 8 to 30 years of age: an estimation of peak bone mass. J Bone Miner Res, 26(8):1729-1739, 2011.

[51] S Bernard, Q Grimal, and P Laugier. Accurate measurement of cortical bone elasticity tensor with resonant ultrasound spectroscopy. Journal of the Mechanical Behavior of Biomedical Materials, 18:12-19, 2013.

[52] L. Peralta, X. Cai, P. Laugier, and Q. Grimal. A critical assessment of the in-vitro measurement of cortical bone stiffness with ultrasound. Ultrasonics, 80:119-126, 2017.

[53] P Laugier and G Ha $\backslash \backslash \mathrm{i}$ at, editors. Bone Quantitative ultrasound. Springer, New York, 2010.

[54] E Camus, M Talmant, G Berger, and P Laugier. Analysis of the axial transmission technique for the assessment of skeletal status. Journal of the Acoustical Society of America, 108:3058-3065, 2000.

[55] Quentin Vallet, Nicolas Bochud, Christine Chappard, Pascal Laugier, and Jean-Gabriel Minonzio. In vivo characterization of cortical bone using guided waves measured by axial transmission. IEEE Trans Ultrason Ferroelectr Freq Control, 2016.

[56] K A Wear. Autocorrelation and cepstral methods for measurement of tibial cortical thickness. Ieee Transactions on Ultrasonics Ferroelectrics and Frequency Control, 50(6):655-660, 2003.

[57] H. Sai, G. Iguchi, T. Tobimatsu, K. Takahashi, T. Otani, K. Horii, I. Mano, I. Nagai, H. Iio, T. Fujita, K. Yoh, and H. Baba. Novel ultrasonic bone densitometry based on two longitudinal waves: Significant 
correlation with pQCT measurement values and age-related changes in trabecular bone density, cortical thickness, and elastic modulus of trabecular bone in a normal Japanese po. Osteoporosis International, 21(10):1781-1790, 2010.

[58] Alexey Tatarinov, Armen Sarvazyan, Gisela Beller, and Dieter Felsenberg. Comparative Examination of Human Proximal Tibiae In Vitro by Ultrasonic Guided Waves and pQCT. Ultrasound in Medicine and Biology, 37(11):1791-1801, 2011.

[59] Petro Moilanen, Patrick H.F. Nicholson, Vantte Kilappa, Sulin Cheng, and Jussi Timonen. Assessment of the cortical bone thickness using ultrasonic guided waves: Modelling and in vitro study. Ultrasound in Medicine \& Biology, 33(2):254-262, feb 2007.

[60] J G Minonzio, M Talmant, and P Laugier. Guided wave phase velocity measurement using multi-emitter and multi-receiver arrays in the axial transmission configuration. Journal of the Acoustical Society of America, 127(5):2913-2919, 2010.

[61] T. Mishima, K. Motoyama, Y. Imanishi, K. Hamamoto, Y. Nagata, S. Yamada, N. Kuriyama, Y. Watanabe, M. Emoto, and M. Inaba. Decreased cortical thickness, as estimated by a newly developed ultrasound device, as a risk for vertebral fracture in type 2 diabetes mellitus patients with eGFR of less than $60 \mathrm{~mL} / \mathrm{min} / 1.73 \mathrm{~m} 2$. Osteoporosis International, 26(1):229-236, 2015.

[62] J. P. Karjalainen, O. Riekkinen, J. Töyräs, M. Hakulinen, H. Kröger, T. Rikkonen, K. Salovaara, and J. S. Jurvelin. Multi-site bone ultrasound measurements in elderly women with and without previous hip fractures. Osteoporosis International, 23(4):1287-1295, 2012.

[63] J. T. Schousboe, O. Riekkinen, and J. Karjalainen. Prediction of hip osteoporosis by DXA using a novel pulse-echo ultrasound device. Osteoporosis International, 28(1):85-93, 2017.

[64] Janina M. Patsch, Andrew J. Burghardt, Samuel P. Yap, Thomas Baum, Ann V. Schwartz, Gabby B. Joseph, and Thomas M. Link. Increased cortical porosity in type 2 diabetic postmenopausal women with fragility fractures. Journal of Bone and Mineral Research, 28(2):313324, 2013. 
[65] R Longo, Q Grimal, P Laugier, S Vanlanduit, and P Guillaume. Simultaneous Determination of Acoustic Velocity and Density of a Cortical Bone Slab: Ultrasonic Model-Based Approach. Ieee Transactions on Ultrasonics Ferroelectrics and Frequency Control, 57(2):496-500, 2010.

[66] Matthieu Loosvelt and Philippe Lasaygues. A Wavelet-Based Processing method for simultaneously determining ultrasonic velocity and material thickness. Ultrasonics, 51(3):325-339, 2011.

[67] Yuriy Tasinkevych, Jerzy Podhajecki, Katarzyna Falińska, and Jerzy Litniewski. Simultaneous estimation of cortical bone thickness and acoustic wave velocity using a multivariable optimization approach: Bone phantom and in-vitro study. Ultrasonics, 65:105-112, 2016.

[68] Josquin Foiret, Jean-Gabriel Minonzio, Christine Chappard, Maryline Talmant, and Pascal Laugier. Combined estimation of thickness and velocities using ultrasound guided waves: a pioneering study on in vitro cortical bone samples. IEEE Trans Ultrason Ferroelectr Freq Control, 61(9):1478-1488, sep 2014.

[69] N. Bochud, Q. Vallet, Y. Bala, H. Follet, J. G. Minonzio, and P. Laugier. Genetic algorithms-based inversion of multimode guided waves for cortical bone characterization. Physics in Medicine and Biology, 61(19):6953-6974, 2016.

[70] J.-G. Minonzio, N. Bochud, Q. Vallet, Y. Bala, D. Ramiandrisoa, H. Follet, D. Mitton, and P. Laugier. Bone cortical thickness and porosity assessment using ultrasonic guided waves: an ex vivo validation study. Bone, submitted, 2018.

[71] Valeria Beltrame, Roberto Stramare, Nicola Rebellato, Federico Angelini, Anna Chiara Frigo, and Leopoldo Rubaltelli. Sonographic evaluation of bone fractures: A reliable alternative in clinical practice? Clinical Imaging, 36(3):203-208, 2012.

[72] R. Zheng, L.H. Le, M.D. Sacchi, and E. Lou. Imaging internal structure of long bones using wave scattering theory. Ultrasound in Medicine and Biology, 41(11), 2015.

[73] G. Renaud, P. Kruizinga, D. Cassereau, and P. Laugier. In vivo ultrasound imaging of the bone cortex. Phys Med Biol, 63(12):125010, Jun 2018. 
[74] P Lasaygues. Assessing the cortical thickness of long bone shafts in children, using two-dimensional ultrasonic diffraction tomography. $\mathrm{Ul}$ trasound in Medicine and Biology, 32(8):1215-1227, 2006.

[75] Simon Bernard, Vadim Monteiller, Dimitri Komatitsch, and Philippe Lasaygues. Ultrasonic computed tomography based on full-waveform inversion for bone quantitative imaging. Physics in Medicine and Biology, 62(17):7011-7035, 2017.

[76] J Y Rho, M C Hobatho, and R B Ashman. Relations of mechanical properties to density and CT numbers in human bone. Med Eng Phys, 17(5):347-55., 1995.

[77] A A Espinoza Orías, J M Deuerling, M D Landrigan, J E Renaud, and $\mathrm{R} K$ Roeder. Anatomic variation in the elastic anisotropy of cortical bone tissue in the human femur. J Mech Behav Biomed Mater, 2(3):255263, 2009.

[78] Quentin Grimal, Guillermo Rus, William J Parnell, and Pascal Laugier. A two-parameter model of the effective elastic tensor for cortical bone. J Biomech, 44(8):1621-1625, 2011.

[79] Ch Hellmich and F J Ulm. can the diverse elastic properties of trabecular and cortical bone be attributed to only a few tissue-independant phase properties and their interactions? Biomechanics and Modeling in Mechanobiology, 2:219-238, 2004.

[80] Mathilde Granke, Quentin Grimal, William J Parnell, Kay Raum, Alf Gerisch, Françoise Peyrin, Amena Saïed, and Pascal Laugier. To what extent can cortical bone millimeter scale elasticity be predicted by a two phase composite model with variable porosity? Acta Biomater, $12: 207-215$, oct 2015 .

[81] William Grimal, Quentin, Parnell. BonHom: program for homogenization.

[82] Elizabeth A Zimmermann, Björn Busse, and Robert O Ritchie. The fracture mechanics of human bone: influence of disease and treatment. Bonekey Rep, 4:743, 2015.

[83] M F Ashby and D R H Jones. Engineering materials 1. Elsevier, Amsterdam, 3rd edition, 2005. 
[84] Caroline Öhman, Massimiliano Baleani, Carla Pani, Fulvia Taddei, Marco Alberghini, Marco Viceconti, and Marco Manfrini. Compressive behaviour of child and adult cortical bone. Bone, 49(4):769-776, oct 2011.

[85] Q Grimal, S Haupert, D Mitton, L Vastel, and P Laugier. Assessment of cortical bone elasticity and strength: Mechanical testing and ultrasound provide complementary data. Medical Engineering $\&$ Physics, 31(9):1140-1147, 2009.

[86] J D Currey. The effect of porosity and mineral content on the Young's modulus of elasticity of compact bone. J Biomech, 21(2):131-139, 1988.

[87] M B Schaffler and D B Burr. Stiffness of compact bone: effects of porosity and density. J Biomech, 21(1):13-16, 1988.

[88] Xiran Cai. Multiscale investigation of the elastic properties of human cortical bone measured by resonant ulrasound spectroscopy. PhD thesis, Sorbonne Université, 2018.

[89] C Baron, M Talmant, and P Laugier. Effect of porosity on effective diagonal stiffness coefficients (cii) and elastic anisotropy of cortical bone at $1 \mathrm{MHz}$ : a finite-difference time domain study. J Acoust Soc Am, 122(3):1810, 2007.

[90] K Raum, I Leguerney, F Chandelier, E Bossy, M Talmant, A Saied, F Peyrin, and P Laugier. Bone microstructure and elastic tissue properties are reflected in QUS axial transmission measurements. Ultrasound in Medicine and Biology, 31(9):1225-35., 2005.

[91] J Y Rho, P Zioupos, J D Currey, and G M Pharr. Microstructural elasticity and regional heterogeneity in human femoral bone of various ages examined by nano-indentation. Journal of Biomechanics, 35(2):18998., 2002.

[92] Chibuzor T. M. Eneh, Jukka Liukkonen, Markus K. H. Malo, Jukka S. Jurvelin, and Juha Töyräs. Inter-individual changes in cortical bone three-dimensional microstructure and elastic coefficient have opposite effects on radial sound speed. The Journal of the Acoustical Society of America, 138(6):3491-3499, 2015.

[93] Christian Hellmich, Jean-Francois Barthelemy, and Luc Dormieux. Mineral-collagen interactions in elasticity of bone ultrastructure - a 
continuum micromechanics approach. European Journal of Mechanics - A/Solids, 23(5):783-810, 2004.

[94] J M Deuerling, W M Yue, A A E Orias, and R K Roeder. Specimenspecific multi-scale model for the anisotropic elastic constants of human cortical bone. Journal of Biomechanics, 42(13):2061-2067, 2009.

[95] S S Mehta, O K Oz, and P P Antich. Bone elasticity and ultrasound velocity are affected by subtle changes in the organic matrix. $J$ Bone Miner Res, 13(1):114-21., 1998.

[96] G Haiat, S Naili, Q Grimal, M Talmant, C Desceliers, and C Soize. Influence of a gradient of material properties on ultrasonic wave propagation in cortical bone: Application to axial transmission. Journal of the Acoustical Society of America, 125(6):4043-4052, 2009.

[97] Cécile Baron. Using the Gradient of Human Cortical Bone Properties to Determine Age-Related Bone Changes Via Ultrasonic Guided Waves. Ultrasound in Medicine and Biology, 38(6):972-981, 2012.

[98] J. Du, G. Iori, and K. Raum. Imaging of cortical pores using ultrasound contrast agents: Phantom and ex vivo studies. In IEEE International Ultrasonics Symposium, IUS, 2017.

[99] Omid Yousefian, R. D. White, Yasamin Karbalaeisadegh, H. T. Banks, and Marie Muller. The effect of pore size and density on ultrasonic attenuation in porous structures with mono-disperse random pore distribution: A two-dimensional in-silico study. JOURNAL OF THE ACOUSTICAL SOCIETY OF AMERICA, 144(2):709-719, AUG 2018. 